\title{
Pinzamiento de arterias hipogástricas. Estrategia de prevención de hemorragia obstétrica en el manejo de placenta previa acreta
}

\author{
Hypogastric arteries clamping: Strategy for obstetric \\ hemorrhage preventing in placenta accrete
}

\author{
Francisco Hernández Salazar,* Luis Tinoco Téllez, ${ }^{\ddagger}$ Ma. Teresa Huerta J Lucas, $\$$ \\ Luis Gerardo Domínguez Carrillo ${ }^{\Uparrow}$
}

\begin{abstract}
Citar como: Hernández SF, Tinoco TL, Huerta JLMT, Domínguez CLG. Pinzamiento de arterias hipogástricas. Estrategia de prevención de hemorragia obstétrica en el manejo de placenta previa acreta. Acta Med Grupo Angeles. 2021; 19 (2): 214-220. https://dx.doi.org/10.35366/100445
\end{abstract}

\section{Resumen}

Objetivo: Presentar una nueva alternativa quirúrgica a fin de disminuir la hemorragia obstétrica en casos de placenta previaacreta, mediante el pinzamiento temporal de arterias hipogástricas, precediendo a la realización de cesárea-histerectomía. Material y métodos: Se atendieron siete pacientes que acudieron a control de embarazo en institución privada de tercer nivel de atención, en quienes mediante ultrasonido obstétrico se detectó placenta previa y acretismo placentario, recabando datos demográficos, clínicos y de diagnóstico. Resultados: En promedio, la edad gestacional en que se realizó la cesáreahisterectomía fue a las 35 semanas, con variación entre las semanas 34.5 y 37 ; en cuanto a sangrado transoperatorio, en cinco instancias fue de $200 \mathrm{~mL}$, un caso con $150 \mathrm{~mL}$ y un caso con $800 \mathrm{~mL}$; los tiempos quirúrgicos variaron de 90 a 106 minutos, en todos los casos se obtuvieron productos vivos con valoración de Apgar y de Silverman-Anderson dentro de lo normal; para todas estas mujeres la estancia hospitalaria fue de dos días. Conclusiones: El pinzamiento temporal (clampeo) de las arterias hipogástricas previo a la cesárea-histerectomía muestra utilidad en la resolución de incidencias de acretismo placentario.

Palabras clave: Acretismo placentario, hemorragia obstétrica, cesárea-histerectomía obstétrica, placenta previa, pinzamiento de arterias hipogástricas.

\section{Abstract}

Objective: To present a new surgical alternative to reduce obstetric hemorrhage in cases of placenta previa-accreta, by means of temporary impingement at the hypogastric arteries, prior to the performance of caesarean-hysterectomy. Material and methods: Seven patients who attended pregnancy control were managed in a third-level care private institution, in whom obstetric ultrasound detected placenta previa accreta, and collecting demographic, clinical and diagnostic data. Results: On average, the gestational age at which the cesarean section-hysterectomy was performed was at 35 weeks with variation between weeks 34.5 and 37 ; in terms of intraoperative bleeding, in five cases it was $200 \mathrm{~mL}$, one case with $150 \mathrm{~mL}$ and one case with $800 \mathrm{~mL}$; surgical times varied from 90 to 106 minutes. In all cases, live products were obtained with Apgar and Silverman-Anderson assessment within the normal ranges; for all patients the hospital stay was two days. Conclusions: Temporary clamping of the hypogastric arteries, prior to caesarean-hysterectomy, shows usefulness in the resolution of cases with placental accreta.

Keywords: Placental accreta, obstetric hemorrhage, obstetric caesarean-hysterectomy, previous placenta, hypogastric arteries clamping.
* Ginecología y Obstetricia. División de Ginecología y Obstetricia. Hospital Clínica Siena. León, Guanajuato, México.

₹ Oncología Quirúrgica. División de Cirugía del Hospital Ángeles León. León, Guanajuato, México.

$\S$ Ginecología y Obstetricia. Hospital Clínica Siena. León, Guanajuato, México.

" Especialista en Medicina de Rehabilitación. Facultad de Medicina de la Universidad de Guanajuato, Campus León. México.

\section{Correspondencia}

Francisco Hernández Salazar

Correo electrónico: dr.francisco.hernandez@gmail.com

Aceptado: 02-09-2020.

www.medigraphic.com/actamedica

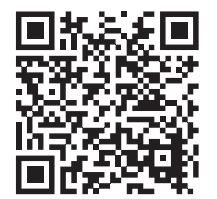




\section{INTRODUCCIÓN}

La placenta previa es causa de hemorragia obstétrica grave, cuando es asociada con acretismo se incrementa la morbimortalidad materna. ${ }^{1}$ En 1995, Lira y colaboradores ${ }^{2}$ reportaron que, ante la existencia de esta condición, surge un riesgo incrementado de espectro de placenta acreta (PAS por sus siglas en inglés) siendo mayor ante edad materna avanzada y con antecedente de dos o más cesáreas.

La placenta previa tiene incidencia de uno por cada 200 a 390 embarazos; en nulíparas es de $0.2 \%$, mientras que en multíparas puede ser mayor al 5\%. El PAS supone $5 \%$ de los casos de acretismo placentario y concentra la mayor morbilidad y mortalidad materna y perinatal. La mortalidad materna alcanza hasta $10 \%$, principalmente por sangrado y sus consecuencias; cuando existe invasión vesical por la placenta, la tasa de mortalidad materna se incrementa hasta $20 \%{ }^{3}$

El reporte de Miller y colegas ${ }^{4}$ indica que la placenta acreta ocurre en $9.3 \%$ de las mujeres con placenta previa y en $0.004 \%$ de las parturientas con placenta normoinserta; a su vez, en las mujeres con placenta previa, con edad mayor o igual a 35 años y antecedente de dos o más cesáreas, el riesgo de placenta acreta se incrementa al 39\% y alcanzó $50 \%$ en mujeres con cuatro cesáreas.

En la actualidad, los factores de riesgo de placenta previa son cada vez más frecuentes, sin embargo, esta patología obstétrica se puede presentar en pacientes sin factores de riesgo, por lo que un control prenatal adecuado permitirá establecer un diagnóstico correcto y ofrecer estrategias de prevención pre y transquirúrgicos. . $^{3,4}$

Para parturientas con placenta previa acreta existen varias alternativas de tratamiento que incluyen: histerectomía obstétrica, ligadura profiláctica de arterias hipogástricas, dejar la placenta in situ para retiro posterior del útero, resección local de placenta, embolización selectiva de arterias hipogástricas, de arterias uterinas y oclusión endovascular con balón de estos vasos. ${ }^{5}$

Con el objetivo de disminuir la hemorragia obstétrica en incidencias de placenta previa-acreta, se ha llevado a cabo la técnica de reducción de flujo circulante hacia el útero mediante pinzamiento temporal (clampeo) de las arterias hipogástricas, como acción anterior a la realización de cesárea-histerectomía en siete pacientes, siendo el motivo de esta comunicación.

\section{MATERIAL Y MÉTODOS}

En estudio retrospectivo, se valoraron siete mujeres que acudieron a control de embarazo en institución privada de tercer nivel de atención, en la ciudad de León, Guanajuato, en las cuales mediante ultrasonido obstétrico se detectó placenta previa y acretismo placentario. En todos los casos se anotaron características demográficas de las pacientes, incluyendo: edad, antecedentes de importancia y gineco-obstétricos, presencia de patologías ginecológicas preexistentes, así como antecedentes de manejo de infertilidad cuando las hubo, control médico prenatal, registro de problemas relacionados y su manejo, edad gestacional de diagnóstico de placenta previa y acretismo, resultados de exámenes de laboratorio preoperatorios, registro de edad gestacional al momento de realizar cesárea-histerectomía, evolución y sangrado transoperatorio, al igual que características del producto incluyendo peso, valoraciones en escalas de Apgar y Silverman-Anderson y, por último, días de estancia hospitalaria. Se anota que, previo a cirugía, se solicitaron en todos los casos seis paquetes globulares y cuatro de plasma fresco congelado como medida de precaución; en todos los sucesos se utilizó colocación de doble vía intravenosa, a su vez se administró cada 15 días, a partir de la semana 26 y hasta la semana 33, un esquema de inductores de madurez pulmonar (acetato de betametasona $2.71 \mathrm{mg}$ y fosfato sódico de betametasona 3 mg en aplicación intramuscular cada 12 horas), además de administración durante todo el embarazo de progesterona natural micronizada, a dosis de 200 mg vía intravaginal cada 24 horas; en los eventos que cursaron con amenaza de parto pretérmino se útero-inhibió con nifedipino cápsulas de 10 mg vía oral cada 8 horas, por el tiempo necesario para lograr este fin. En las siete se procedió a pinzamiento temporal de las arterias hipogástricas de manera bilateral con anterioridad a la realización de cesárea-histerectomía, cuantificando los lapsos quirúrgicos desde incisión en piel hasta el cierre de la misma; por último se registraron los resultados de estudio histopatológico.

\section{Descripción de técnica quirúrgica}

Bajo bloqueo peridural y relajación profunda, paciente en decúbito dorsal, asepsia y antisepsia y colocación de campos estériles se procede a: 1) realizar incisión media supra e infraumbilical, efectuando disección por planos; 2) se exterioriza el útero con producto del embarazo in situ, colocando dos compresas en ambos espacios parietocólicos buscando mantener el intestino fuera de la pelvis (anotando que debe existir buena relajación muscular); 3) se procede a localizar por medio de palpación los uréteres y las arterias iliacas, así como su bifurcación que va a estar situada aproximadamente a 2 o $3 \mathrm{~cm}$ por delante del promontorio (el cirujano debe situarse en el lado opuesto a la arteria que se va pinzar); 4) se realiza una incisión de 3 a 4 cm en el peritoneo posterior (acceso al espacio retroperitoneal) exterior al uréter y medialmente a la arteria hipogástrica; 5) en el lado izquierdo es necesario movilizar y rechazar 
eventualmente el recto sigmoides para mejor exposición de la bifurcación de la arteria iliaca común y poder así localizar a la arteria hipogástrica; 6) se efectúa disección digital y gentil hasta visualizar la arteria iliaca común y su bifurcación, igual que los vasos venosos que se observan de coloración azul por debajo de los vasos bifurcados; 7) después de haber disecado el tejido areolar que recubre la arteria hipogástrica en su cara anterior y lateral, se procede a disecar el tejido que une a esta arteria con la vena iliaca interna, realizando disección con apertura y cierre de la pinza Mixter, hasta ver aparecer la punta de la misma en la cara opuesta; completamente aislada la arteria hipogástrica, en al menos $1 \mathrm{~cm}$ de distancia, esto en los primeros $5 \mathrm{~cm}$ después de la bifurcación de la arteria iliaca común; 8) inmediatos a la bifurcación, se refiere la arteria hipogástrica con una cinta de silicón (Silastic) y se efectúa la misma maniobra en lado contralateral; 9) una vez referidas ambas arterias iliacas internas (y listo el equipo de ginecología y obstetricia y pediatría), se pinzan cada arteria con una pinza de tipo Bulldog curvo de $50 \mathrm{~mm} / 12 \mathrm{~mm}$; 10) inmediatamente después, se incide cuerpo y fondo uterino con incisión corporal clásica tipo Beck, efectuando la extracción del producto; se deja pinzado el cordón umbilical y se deja en su sitio la placenta, sin administración de oxitocina; 11) se afrontan bordes uterinos con pinzas de Allis-Adair y se continúa con el procedimiento de histerectomía clásica de Richardson en un campo exangüe mediante pinzamiento, corte y atadura de ligamentos redondos, anchos, arterias del útero, ligamentos de Mackenrodt, ligamentos útero-sacros; se incide en la parte superior de la vagina con corte transversal de la misma, extrayendo el útero completo; cierre de cúpula vaginal con sutura de vicryl del número uno; 12) una vez finalizada la hemostasia y verificación de la misma se retiran las pinzas de las arterias hipogástricas y se verifica de nuevo el estado de hemostasia; se peritoniza cúpula vaginal, cierre de pared abdominal por planos con técnica convencional y se da por terminado el procedimiento.

\section{RESULTADOS}

El universo estudiado correspondió a siete parturientas (Tabla 1) con edad promedio de 31 años y variación entre 24 y 42 años; en tres casos existieron antecedentes de importancia, siendo tabaquismo, diabetes mellitus en abuela paterna y antecedente de hepatitis tipo A respectivamente; en dos de ellos existió el antecedente de patología preexistente, un caso con obstrucción tubaria bilateral y el otro con exéresis de endometriomas; una paciente logró su embarazo mediante fertilización in vitro y transferencia embrionaria, y otra por inducción de ovulación e inseminación artificial; en cuanto al número de gestaciones, de forma respectiva correspondió a dos mujeres con tres, dos con dos y tres con la gestación motivo de consulta; tres de ellas presentaron antecedente de cesáreas anteriores, correspondiendo dos en un caso y uno en dos pacientes respectivamente; se reportó sólo un caso con embarazo gemelar; en la totalidad de casos existió control prenatal; en cinco de ellos se presentó cuadro de amenaza de parto prematuro entre las semanas 24 a 30 de gestación, la cual fue manejada con útero-inhibidores; el diagnóstico de placenta previa y acretismo se realizó entre la semanas 21 a 35, existiendo un caso con diagnóstico en la semana 16; en general los exámenes de laboratorio preoperatorios se encontraron dentro de lo normal, excepto el caso de la paciente con embarazo gemelar, quien cursó con anemia; en promedio, la edad gestacional en que se realizó la cesárea-histerectomía fue a las 35 semanas con variación entre las semanas 34.5 y 37 ; en cuanto a sangrado transoperatorio, en cinco casos fue de $200 \mathrm{~mL}$, un caso con 150 $\mathrm{mL}$ y un caso con $800 \mathrm{~mL}$ (se anota que éste correspondió a la paciente número siete que presentó histopatológicamente placenta increta); los tiempos quirúrgicos variaron de 90 a 106 minutos; en todos los eventos se obtuvieron productos vivos con valoraciones de Apgar y de SilvermanAnderson dentro de lo normal; la estancia hospitalaria fue de dos días para todas las mujeres; no se observaron complicaciones postoperatorias. Los diagnósticos histopatológicos se muestran en la Tabla 2, correspondiendo un caso a placenta increta, cuatro casos a placenta previa y acretismo y dos más con inserción baja de placenta, una abarcando al cérvix y ambas con acretismo.

\section{DISCUSIÓN}

Desde el punto de vista fisiopatogénico, la llamada cama decidual, que se forma durante la gestación y que consta en su origen de tejido citotrofoblástico, juega un papel importante cuando se trata de hemorragia obstétrica, ya que la adhesión de la placenta (acreta, increta y percreta) incluye: a) implantación anormal al miometrio debido al desarrollo anómalo de la capa de Nitabuch, y b) la ausencia parcial o total de la decidua basal incrementa la invasividad del citotrofoblasto que habitualmente se asocia con alteraciones en la angiogénesis trofoblástica. Resulta ser el primer punto el más frecuente cuando este tejido se implanta en un miometrio con cicatrices (cesáreas, legrados, miomectomías), confiriendo un riesgo tres veces mayor de padecer acretismo placentario; al respecto, el antecedente de dos cirugías anteriores incrementa el riesgo de 8.6 a 29.8\%; esto condiciona una falta de separación entre los planos deciduales al miometrio quedando fijos algunos cotiledones placentarios, esta situación es más evidente durante el proceso de alumbramiento, pues trae consigo la apertura de los vasos espirales que sangran profusamente, 
Tabla 1: Datos demográficos, clínicos y diagnósticos en siete pacientes con placenta acreta atendidas con pinzamiento de arterias hipogástricas previo a cesárea/histerectomía.

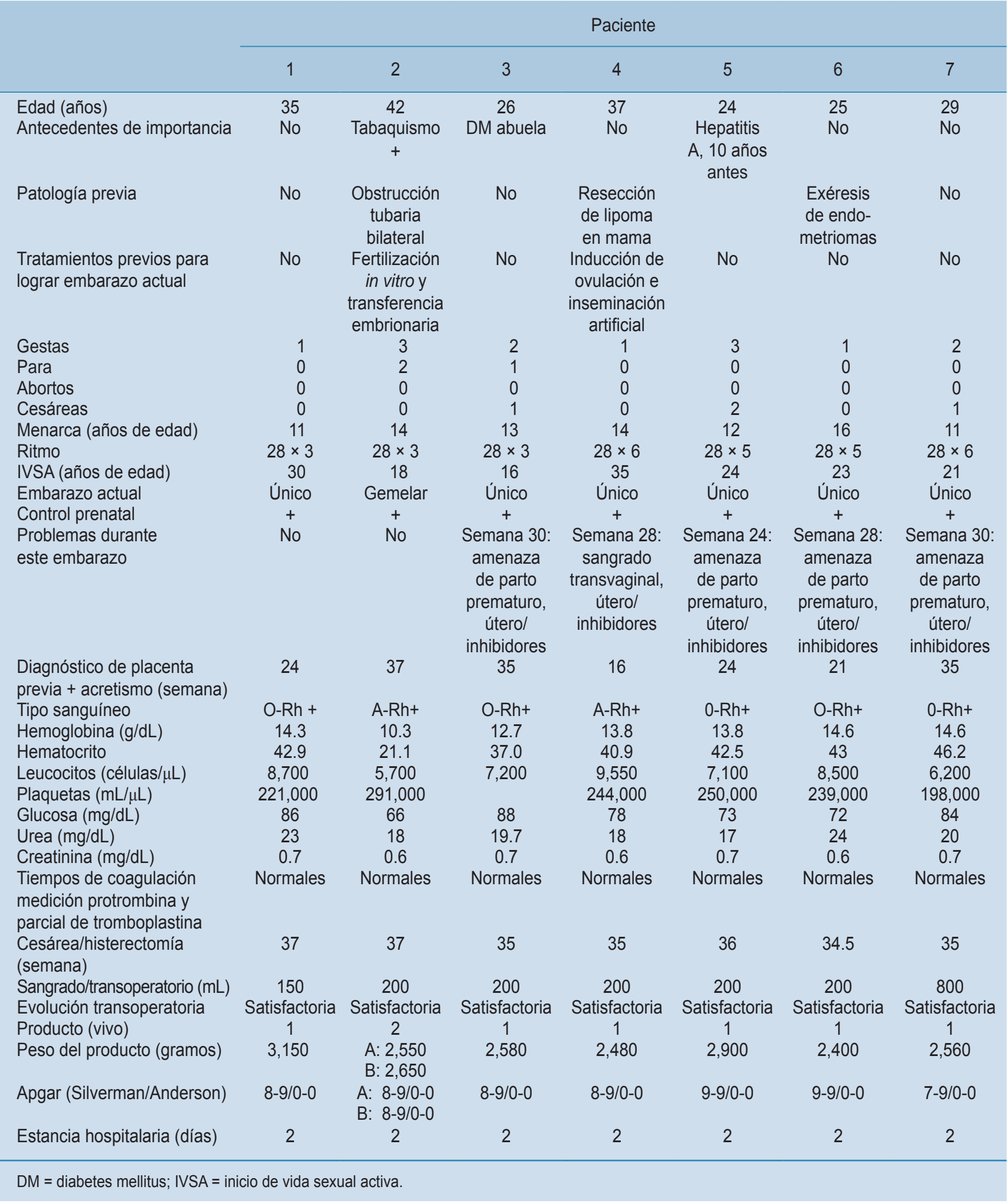


Tabla 2: Diagnósticos histopatológicos en siete pacientes con acretismo placentario.

Paciente Diagnóstico histopatológico

$1 \quad$ Placenta previa central marginal posterior con acretismo placentario

$2 \quad$ Placenta previa central total con acretismo

3 Placenta previa central total con acretismo

4 Placenta de inserción inferior y acreta, que afecta tercio interno del miometrio, edema del estroma cervical e infarto vascular placentario reciente

5 Placenta acreta que involucra el tercio inferior de pared uterina, sin afectar cérvix, involucra un $50 \%$ de miometrio, necrosis isquémica focal con áreas de hemorragia en tejido placentario

6 Inserción placentaria baja con acretismo, con borde marginal cubriendo la totalidad del cérvix

$7 \quad$ Placenta acreta que involucra $2 / 3$ inferiores de la pared uterina sin afectación del cérvix, abarcando la totalidad de pared miometrial sin afectación serosa, necrosis isquémica focal con áreas de hemorragia

alterando los mecanismos hemostáticos y hemodinámicos, incrementando a su vez el riesgo de sangrado obstétrico, relacionados con la disminución de endostatina e hipoxia, de acuerdo con el estudio de Bischof y colegas. ${ }^{6}$ Lo segundo está asociado con una amplia gama de factores, como endometritis, fertilización asistida, edad mayor a 35 años y uso de cocaína.

Hoy en día, el método diagnóstico que logra detectar placenta previa con acretismo es el ultrasonido; entre los diversos signos hacia su diagnóstico ultrasonográfico destacan: 1) pérdida de la zona hipoecoica que normalmente se encuentra entre la placenta y el endometrio; 2) espacios vasculares lacunares en el parénquima placentario; 3) pérdida de la interfaz entre la pared vesical y la serosa uterina; 4) vascularidad aumentada en el piso pélvico, y 5) rara vez puede verse tejido placentario más allá de la serosa del útero, anotando que el Doppler color y Doppler ayudan a identificar la invasión placentaria. ${ }^{7}$

La hemorragia obstétrica puede causar pérdida masiva de sangre, lo suficientemente rápida como para ser fatal, este evento es esperado en pacientes que sufren de placenta previa central con acretismo, siendo una causa de histerectomía obstétrica; dicha cirugía de alta morbilidad y mortalidad materna implica un alto riesgo de sangrado y complicaciones hematológicas asociadas por la coagulación intravascular diseminada. Se han hecho esfuerzos procurando evitar o diferir la histerectomía hasta el momento en el que se aminoren las condiciones agravantes, este manejo llamado "conservador" consiste precisamente en retrasar la histerectomía a través de medios físicos y químicos hasta que mejoren las condiciones vasculares del útero y permitan reducir la morbilidad por hemorragia. Kably y asociados $^{8}$ reportan tres casos de manejo conservador, practicando la cesárea sin remover la placenta, efectuando histerorrafia y manejo expectante con la placenta in situ, aunado a la aplicación de metotrexato intravascular, a través de un catéter femoral que transcurre por los vasos uterinos, a fin de que el fármaco llegue directamente a la placenta. Posterior a la aplicación del mismo, se lleva a cabo el procedimiento de embolización de arterias uterinas, seleccionando los vasos nutricios de la placenta apoyados por medio de fluoroscopia, con el fin de provocar la reducción y/o expulsión posterior de la placenta.

Estas propuestas tienen en común inducir la regresión del tejido placentario invasor y la vascularidad pélvica antes de proceder a la cirugía definitiva, para este propósito, una vez extraído el neonato, se ha optado por dejar la placenta en la cavidad uterina y durante el transcurso de las semanas se ha ensayado a administrar metotrexato por diversas vías: oral, intravenosa, intramuscular e intraplacentaria, con el propósito de lograr la regresión del tejido placentario ("técnica Rodríguez Bosch"). ${ }^{9}$ Por otra parte, un metaanálisis publicado por Clausen ${ }^{5}$ señala que el manejo conservador, en el sentido de dejar la placenta in situ, buscando su resorción espontanea, parece estar asociado con mayores complicaciones a largo plazo; de las pacientes en que se dejó la placenta in situ, 61\% sufrió al menos una complicación postoperatoria (hemorragia e infección), comparada con los casos en los que se efectuó la resección transoperatoria del útero. Se ha reportado la realización de histerectomía obstétrica de forma convencional y, al momento de ligar arterias del útero al producto del embarazo sin traccionar placenta, dejándola en su sitio y luego continuar con la histerectomía obstétrica.

Se ha publicado el uso de embolización selectiva de arterias de útero, ${ }^{10}$ con el que se logra la eliminación espontánea gradual y total del tejido placentario, sin requerir tratamiento adicional, al igual que la embolización de arterias uterinas o hipogástricas inmediatamente después de la extracción del producto; sin embargo, la disponibilidad 
de un radiólogo intervencionista con experiencia en esta clase de procedimientos no es común.

La ligadura de arterias hipogástricas fue descrita por Kelly en 1894, quien al parecer fue el primero en realizar y relatar la intervención, tomando tanta importancia en el manejo de la hemorragia postparto que no en vano se describe que debe realizarse de forma profiláctica o en causas de sangrados incoercibles, por rotura uterina, útero de Couvelaire, traumatismos del canal del parto y trastornos adherenciales de la placenta, pues la ligadura referida reduce el flujo sanguíneo del útero hasta en un $80 \%{ }^{11-13}$ por la disminución en la presión del pulso, transformando un sistema arterial en un sistema venoso, el cual disminuye el flujo sanguíneo y la presión intravascular, con la consiguiente disminución de la pérdida sanguínea, ${ }^{14}$ de ahí que el Colegio Americano de Ginecología y Obstetricia (ACOG por sus siglas en inglés) recomienda la ligadura de arterias hipogástricas como un procedimiento útil en el tratamiento de la hemorragia postparto. ${ }^{15}$ No obstante, es una técnica que conlleva complicaciones, tales como: áreas de isquemia o necrosis sobre el piso pélvico, dificultad de micción en las primeras horas del postoperatorio supuestamente por trastornos transitorios en la circulación de la vejiga; fístulas urinarias, fundamentalmente relacionadas con la cirugía radical, ligadura incompleta, daño ureteral, lesión de la vena hipogástrica o sangrado continuo por la circulación colateral, lesión a nervios del plexo hipogástrico y claudicación. Algunos autores sostienen la hipótesis de que posterior a la ligadura de las arterias hipogástricas, la circulación colateral puede ser adecuada en condiciones normales, pero en extremo desastrosas en situaciones de estrés o en casos de mujeres ancianas con ateroesclerosis. ${ }^{16}$ Por otra parte, el reporte de Kuhn y su equipo ${ }^{17}$ no mostró beneficios con respecto a la cantidad de pérdida de sangre y unidades transfundidas con el empleo profiláctico de ligadura de arterias hipogástricas, al compararlo con histerectomía obstétrica en parturientas con placenta previa percreta.

Para contrastar los resultados de ese estudio con algunos reportados en la literatura se tiene: el reporte de Yildiz y colaboradores $^{18}$ en 24 pacientes, 15 con placenta previa (el doble de los casos de este reporte) y nueve con atonía uterina, reportan resultados semejantes tanto en lapsos quirúrgicos como cantidad de sangrado, no existiendo muertes maternas ni fetales; el reporte de Morel y colaboradores ${ }^{19}$ menciona que en caso de sangrado severo postparto el procedimiento es inmediatamente efectivo en $80 \%$ de las ocasiones, al igual que lo avalan los reportes de Simsek y colegas ${ }^{20}$ y de Unal O y asociados; ${ }^{21}$ por otra parte, el único estudio que no concuerda en sus resultados con el resto de los confrontados incluyendo el presente, pertenece a Iwata y colegas, ${ }^{22}$ quienes indican que en 23 incidencias de histerectomía por placenta acreta, efectuada con y sin ligadura temporal de arterias hipogástricas, no encontraron diferencias entre cantidad de sangrado, tiempos quirúrgicos y días de hospitalización, por lo que consideran no importante el realizar la ligadura.

No obstante el número pequeño de casos, los resultados del presente trabajo, al pinzar temporalmente las arterias hipogástricas con anterioridad a la realización de cesárea-histerectomía, muestra ventajas al compararla con las otras técnicas mencionadas, como es la oclusión intravascular de dichos vasos, sobre todo cuando no se cuenta con un servicio de radiología intervencionista, siendo además una técnica reversible que no generó complicaciones, por restituirse la circulación tisular dependiente de ellas, por lo que puede considerarse dentro de las "prácticas que salvan vidas" y, de acuerdo con Sziller ${ }^{23}$ y su grupo, el procedimiento inicialmente se utilizó en cirugía a fines del siglo XIX intentando controlar la hemorragia intratable del útero de mujeres con cáncer avanzado, logrando su efectividad evitar la muerte por sangrado en casos de placenta acreta, lo cual hace que se considere un procedimiento efectivo, que debe ser enseñado durante el entrenamiento de la residencia de ginecología y obstetricia, resultados que se reflejan en el pequeño número de ocasiones en este reporte, sin embargo, al no contar con un grupo control, implica la posibilidad de sesgos, siendo una desventaja del trabajo presentado, no obstante, el difundir estas técnicas ayudará a disminuir la morbimortalidad del problema.

\section{CONCLUSIONES}

El pinzamiento de arterias hipogástricas, anterior a la realización de cesárea histerectomía es una estrategia eficaz para reducir los riesgos de hemorragia obstétrica y sus consecuencias.

\section{REFERENCIAS}

1. Medina LJM, Hernández EAI. Placenta previa acreta: un problema singular. Comunicación de dos casos y revisión de la bibliografía. Ginecol Obstet Mex. 2006; 74 (11): 599-604.

2. Lira Plascencia J, Ibargüengoitia Ochoa F, Argueta Zúñiga M, Karchmer S. Placenta praevia/accreta and previous cesarean section. Experience of five years at the Mexico National Institute of Perinatalogy. Ginecol Obstet Mex. 1995; 63: 337-340. PMID: 7672649.

3. Hernández-Ojeda H, Torres-Hernández RM, Rivera Hernández JO. Acretismo placentario con placenta previa. Reporte de un caso. Ginecol Obstet Mex. 2014; 82 (8): 552-557.

4. Miller DA, Chollet JA, Goodwin TM. Clinical risk factors for placenta previa-placenta accreta. Am J Obstet Gynecol. 1997; 177 (1): 210214. doi: 10.1016/s0002-9378(97)70463-0.

5. Clausen C, Lonn L, Langhoff-Roos J. Management of placenta percreta: a review of published cases. Acta Obstet Gynecol Scand. 2014; 93 (2): 138-143. doi: 10.1111/aogs.12295. 
6. Bischof P, Meisser A, Campana A. Paracrine and autocrine regulators of trophoblast invasion--a review. Placenta. 2000; 21 Suppl A: S55-S60. doi: 10.1053/plac.2000.0521.

7. Haghenbeck-Altamirano FJ, Leis-Márquez T, Ayala-Yáñez R, JuárezGarcía LC, García-Moreno C. Diagnóstico antenatal de acretismopercretismo placentario. Ginecol Obstet Mex. 2013; 81: 259-271.

8. Kably AA, Acosta MM, Bustamante QY, Sánchez VGE, Lozano ZH, Pérez MJA. Tratamiento conservador del acretismo placentario. Reporte de tres casos y revisión de la literatura. Acta Med. 2018; 16 (1): $72-75$.

9. Rodríguez-Bosch M, Dueñas-García O. Hemorragia obstétrica: técnica Rodríguez-Bosch para el tratamiento de la placenta adherida. México: Nieto Editores; 2016. pp. 62-72.

10. Weinstein A, Chandra P, Schiavello H, Fleischer A. Conservative management of placenta previa percreta in a Jehovah's Witness. Obstet Gynecol. 2005; 105 (5 Pt 2): 1247-1250. doi: 10.1097/01. AOG.0000157762.65732.1b.

11. Espitia de la Hoz FJ, Zuluaga Cortés OE, Orozco Santiago L. Ligadura de arterias hipogástricas en hemorragia postparto severa. CES Med. 2016; 30 (1): 26-34.

12. Oyelese Y, Scorza WE, Mastrolia R, Smulian JC. Postpartum hemorrhage. Obstet Gynecol Clin North Am. 2007; 34: 421-441.

13. Chelli D, Boudaya F, Dimassi K, Gharbi B, Najjar I, Sfar E et al. Hypogastric artery ligation for post-partum hemorrhage. J Gynecol Obstet Biol Reprod (Paris). 2010; 39 (1): 43-49. doi: 10.1016/j. jgyn.2009.10.002.

14. Hurtado EG, Cárdenas GS, Barrón FA, Cordero GCA, Morales PM, Vargas HVM et al. Ligadura de arterias hipogástricas, una cirugía heroica para evitar la mortalidad en ginecoobstetricia. Arch Inv Mat Inf. 2009; 1 (3): 138-143.

15. ACOG educational bulletin. Postpartum hemorrhage. Number 243, January 1998 (replaces No. 143, July 1990). American College of Obstetricians and Gynecologists. Int J Gynaecol Obstet. 1998; 61 (1): 79-86. PMID: 9622181.
16. Bautista-Gómez E, Morales-García V, Hernández-Cuevas J, Calvo Aguilar O, Flores-Romero AL, Santos-Pérez U. Una alternativa quirúrgica para acretismo placentario. Ginecol Obstet Mex. 2011; 79 (5): 298-302.

17. Kuhn T, Martimucci K, Al-Khan A, Bilinski R, Zamudio S, AlvarezPerez J. Prophylactic hypogastric artery ligation during placenta percreta surgery: a retrospective cohort study. AJP Rep. 2018; 8 (2): e142-e145. doi: 10.1055/s-0038-1666793.

18. Yildiz C, Akkar OB, Karakus S, Cetin A, Yanik A. Hypogastric artery ligation for obstetrical hemorrhage: clinical experience in a tertiary care center. Turk J Med Sci. 2015; 45 (6): 1312-1316.

19. Morel O, Malartic C, Muhlstein J, Gayat E, Judlin P, Soyer P et al. Pelvic arterial ligations for severe post-partum hemorrhage. Indications and techniques. J Visc Surg. 2011; 148 (2): e95-102.

20. Yavuz S, Ercan Y, Ebru C, Ilgin T, Abdullah K, Ugur T et al. Efficacy of internal iliac artery ligation on management of postpartum hemorrhage and its impact on the ovarian reserve. J Turk Soc Obstet Gynecol. 2012; 9 (3): 153-158.

21. Unal O, Kars B, Buyukbayrak EE, Karsidag AY, Turan C. The effectiveness of bilateral hypogastric artery ligation for obstetric hemorrhage in three different underlying conditions and its impact on future fertility. J Matern Fetal Neonatal Med. 2011; 24 (10): 1273-1276.

22. Iwata A, Murayama Y, Itakura A, Baba K, Seki H, Takeda S. Limitations of internal iliac artery ligation for the reduction of intraoperative hemorrhage during cesarean hysterectomy in cases of placenta previa accreta. J Obstet Gynaecol Res. 2010; 36 (2): 254-259.

23. Sziller I, Hupuczi P, Papp Z. Hypogastric artery ligation for severe hemorrhage in obstetric patients. J Perinat Med. 2007; 35 (3): 187-192.

Financiamiento y conflicto de intereses: Los autores declaran no tener conflicto de intereses, además aclaran no haber recibido ningún tipo de financiamiento en la elaboración del manuscrito. 